\title{
Final-state interactions in hypernuclear decay
}

\author{
A. Parreño* \\ Institute for Nuclear Theory, University of Washington, Seattle, Washington 98195-1550 \\ A. Ramos \\ Departament d'Estructura i Constituents de la Matèria, Universitat de Barcelona, Diagonal 647, E-08028 Barcelona, Spain
}

(Received 27 April 2001; published 6 December 2001)

\begin{abstract}
We present an update of the one-meson-exchange (OME) results for the weak decay of $s$ - and $p$-shell hypernuclei [A. Parreño, A. Ramos, and C. Bennhold, Phys. Rev. C 56, 339 (1997)], paying special attention to the role played by final state interactions between the emitted nucleons. The present study also corrects for a mistake in the inclusion of the $K$ and $K^{*}$ exchange mechanisms, which substantially increases the ratio of neutron-induced to proton-induced transitions $\Gamma_{n} / \Gamma_{p}$. With the most up-to-date model ingredients, we find that the OME approach is able to describe very satisfactorily most of the measured observables, including the ratio $\Gamma_{n} / \Gamma_{p}$.
\end{abstract}

DOI: 10.1103/PhysRevC.65.015204

PACS number(s): 21.80.+a, 13.30.Eg, 13.75.Ev, 13.75.Cs

\section{INTRODUCTION}

Hypernuclei are bound systems of nonstrange and strange baryons. In present facilities, hypernuclei are created with hadronic reactions-such as $(\pi, K)$ at Brookhaven and KEK-or electroproduction ones-such as $\left(e, e^{\prime} K\right)$ at TJNAF. The decay of those objects proceeds via the weak interaction which is 10 orders of magnitude slower than the strong one and violates parity, isospin, and strangeness. For the very light hypernuclei the mesonic decay mode $(\Lambda$ $\rightarrow \pi N$ ) is dominant, but as $A$ increases so does the Pauli blocking acting on the outgoing nucleon, and hypernuclei mainly decay via the one-nucleon induced nonmesonic mode, $\Lambda N \rightarrow N N$. Since the pioneering phenomenological model of Block and Dalitz [1], many approaches have been developed to understand the dynamics of the decay, and the results have been collected in extensive review articles [2-4]. Many works are based on a meson exchange picture, either using a simple one-pion-exchange mechanism [5,6], or also including heavier meson exchanges, such as the $\rho$ [7] or the complete pseudoscalar and vector meson octets $[8,9]$. The effect of correlated-two pion exchange mechanism [1012] as well as the role of $\Delta I=3 / 2$ transitions, implemented in a meson-exchange picture [13], have also been investigated. A four quark weak transition effective Hamiltonian, corrected by QCD, which contains both $\Delta I=1 / 2$ and $3 / 2$ transitions, has also been applied in the study of the weak decay of hypernuclei [14-17]. In general, the weak decay rates are reasonably reproduced by the models but the ratio of neutron induced $(\Lambda n \rightarrow n n)$ to proton induced $(\Lambda p \rightarrow n p)$ decays $\Gamma_{n} / \Gamma_{p}$ turns out to be smaller than the experimental value, which is of the order of 1 or larger [18-20], although new recent theoretical progress has been achieved into the solution of this puzzle $[12,16]$. In addition to $\Lambda N \rightarrow N N$, the decay can also proceed via the two-nucleon induced process

\footnotetext{
*Present address: Departament d'Estructura i Constituents de la Matèria, Universitat de Barcelona, Diagonal 647, E-08028 Barcelona, Spain.
}

$\Lambda N N \rightarrow N N N$ originally studied in Ref. [21], which amounts to about $15 \%$ of the total width [22-24] and cannot be neglected in the experimental analysis trying to extract the ratio $\Gamma_{n} / \Gamma_{p}$.

The high momentum of about $400 \mathrm{MeV} / c$ transferred in the $\Lambda N \rightarrow N N$ reaction makes this process quite sensitive to short range physics. As a consequence, the strong baryonbaryon interaction both in the initial and final states plays a quite important role. The purpose of the present work is to revisit the one-meson-exchange model of Ref. [8], with an especial interest in quantifying the effect of final state interactions, as well as the uncertainties of different model ingredients. In doing so, we will also point out a sign error encountered in certain transitions mediated by the exchange of strange mesons which, when corrected, gives rise to a considerably increase in the $\Gamma_{n} / \Gamma_{p}$ ratio. We will analyze the sensitivity of our results to the way final state interactions of the emitted nucleons are implemented, using different prescriptions to obtain the $N N$ scattered wave function. Our study concludes that with the appropriate treatment of final state interactions and with the correct sign for the contribution of the strange mesons, the one-meson-exchange model is able to describe very satisfactorily most of the measured observables, including the elusive ratio $\Gamma_{n} / \Gamma_{p}$.

\section{WEAK TRANSITION POTENTIAL}

The weak transition potential is obtained by following the model of Ref. [8]. In analogy to one-boson-exchange (OBE) based models of the strong interaction, the present formalism includes not only the exchange of the long-ranged pion, but also more massive mesons which account for shorter distances. This potential has been presented in previous papers and, therefore, it is not going to be discussed here in a great detail. However, we would like to show its expression in coordinate space, which represents a compact way of including all the mesons and transition channels in the mechanism. The $\vec{r}$-space potential then reads 


$$
V(\vec{r})=\sum_{i} \sum_{\alpha} V_{\alpha}^{(i)}(\vec{r})=\sum_{i} \sum_{\alpha} V_{\alpha}^{(i)}(r) \hat{O}_{\alpha}(\hat{r}) \hat{I}^{(i)},
$$

where the index $i$ runs over the different mesons exchanged
( $i=1, \ldots, 6$ represents $\left.\pi, \eta, K, \rho, \omega, K^{*}\right)$ and $\alpha$ over the different spin operators: central spin independent $(C)$, central spin dependent (SS), tensor $(T)$, and parity violating (PV). The angular dependence is represented by the $\hat{O}_{\alpha}(\hat{r})$ operator, explicitly given by

$$
\hat{O}_{\alpha}(\hat{r})=\left\{\begin{array}{l}
\hat{1}, \quad C \text { (only for vector mesons), } \\
\vec{\sigma}_{1} \vec{\sigma}_{2}, \quad \mathrm{SS}, \\
S_{12}(\hat{r})=3 \vec{\sigma}_{1} \hat{r} \vec{\sigma}_{2} \hat{r}-\vec{\sigma}_{1} \vec{\sigma}_{2}, \quad T, \\
i \vec{\sigma}_{2} \hat{r}, \quad \mathrm{PV} \text { (for pseudoscalar mesons), } \\
{\left[\vec{\sigma}_{1} \times \vec{\sigma}_{2}\right] \hat{r}, \quad \mathrm{PV} \text { (for vector mesons), }}
\end{array}\right.
$$

while the isospin operator $\hat{I}^{(i)}$ takes the form $\vec{\tau}_{1} \vec{\tau}_{2}$ for isovector mesons $(\pi, \rho), \hat{1}$ for isoscalar mesons $(\eta, \omega)$, and a combination of both operators for the isodoublet $\left(K, K^{*}\right)$.

The different pieces of the potential $V_{\alpha}^{(i)}(r)$ are found by Fourier transforming the potential in momentum space. In particular, for pseudoscalar mesons the $\vec{q}$-space potential reads

$$
\begin{aligned}
V_{p s}{ }^{(i)}(\vec{q})= & -G_{F} m_{\pi}^{2} \frac{g_{\mathrm{BB} \mathrm{M}^{(i)}}}{2 M_{S}}\left(A^{(i)}+\frac{B^{(i)}}{2 M_{W}} \vec{\sigma}_{1} \vec{q}\right) \\
& \times \frac{\vec{\sigma}_{2} \vec{q}}{\vec{q}^{2}+\mu^{(i) 2}} \hat{I}^{(i)},
\end{aligned}
$$

where $G_{F} m_{\pi}^{2}=2.21 \times 10^{-7}$ is the Fermi weak constant times the pion mass squared, $g_{\mathrm{BB}} \mathrm{M}^{(i)}$ the coupling at the strong baryon-baryon-meson $\left(\mathrm{BBM}^{(i)}\right)$ vertex, $A^{(i)}$ and $B^{(i)}$ the parity violating $(\mathrm{PV})$ and parity conserving $(\mathrm{PC})$ weak couplings, respectively, $\mu^{(i)}$ the meson mass, and $M_{S}\left(M_{W}\right)$ the average of the baryon masses at the strong (weak) vertex.

We want to note here that the convention has always been to direct the momentum towards the strong vertex and, in connection to this, care must be taken when this expression is applied to $K\left(K^{*}\right)$ exchange. In practice, the combination with the other nonstrange meson contributions requires to exchange $\vec{q}$ by $-\vec{q}$ and the subindices $1 \leftrightarrow 2$ in the amplitudes involving strange meson exchange. In this way, the momentum transfer $\vec{q}$ will always be emitted at the $\Lambda \mathrm{NM}$ vertex, independently of whether it is weak or strong.

Related to the above comment, we note that the combination of nonstrange meson amplitudes with strange meson ones was not properly done in Ref. [8]. In that work, as in the present one, the transition amplitudes are decomposed in partial wave contributions of definite spin and isospin. In the case of $s$-shell hypernuclei, these amplitudes read [1]

$$
\begin{array}{ll}
a:{ }^{1} S_{0} \rightarrow{ }^{1} S_{0} & (I=1), \\
b:{ }^{1} S_{0} \rightarrow{ }^{3} P_{0} & (I=1), \\
c:{ }^{3} S_{1} \rightarrow{ }^{3} S_{1} & (I=0),
\end{array}
$$

$$
\begin{aligned}
& d:{ }^{3} S_{1} \rightarrow{ }^{3} D_{1} \quad(I=0), \\
& e:{ }^{3} S_{1} \rightarrow{ }^{1} P_{1} \quad(I=0), \\
& f:{ }^{3} S_{1} \rightarrow{ }^{3} P_{1} \quad(I=1) .
\end{aligned}
$$

As it has also been noted in Ref. [16], the mistake in the calculation of the strange meson amplitudes of Ref. [8] is corrected by changing the sign of the amplitude $f:{ }^{3} S_{1}$ $\rightarrow{ }^{3} P_{1}$. More generally, it can be easily shown that one must add the phase factor

$$
(-1)^{L_{0}+L+S_{0}+S} \times(-1)^{T_{0}-t_{N}-t_{\Lambda}} \times(-1)^{T-t_{1}-t_{2}}
$$

to each of the strange meson amplitudes calculated in Ref. [8], where $L_{0}$ is the initial $\Lambda N$ relative orbital angular momentum $\left(L_{0}=0\right.$ for $s$-shell hypernuclei and $L_{0}=0,1$ for $p$-shell hypernuclei), $L$ the final $N N$ one, $S_{0}$ the initial twobody spin, $S$ the final one, $T_{0}$ the initial $\Lambda N$ isospin, and $T$ the final $N N$ one. The symbols $t_{N}, t_{1}, t_{2}$ stand for the nucleon isospin value of $1 / 2$, while $t_{\Lambda}$ is the $\Lambda$ one. Note that the $\Delta I=1 / 2$ rule is imposed by dressing the $\Lambda$ with isospin $1 / 2$. Therefore, $T_{0}$ can take the values 0 and 1 . If we accept violations of this rule and consider $\Delta I=3 / 2$ transitions in the potential, the $\Lambda$ will be dressed with $t_{\Lambda}=3 / 2$ and $T_{0}$ will take the values 1 and 2 . In any case, once we dress the $\Lambda$ with isospin $\neq 0$, we can use the same isospin formalism as in the strong sector and work as if isospin was conserved through the transition. Since the final $N N$ pair has total isospin $T=0$ and 1 , and $T_{0}=T$, we will have

$$
T_{0}= \begin{cases}0,1 & \text { for } \Delta I=1 / 2 \\ 1 & \text { for } \Delta I=3 / 2 .\end{cases}
$$

This produces an overall factor $\pm(-1)^{L_{0}+L+S_{0}+S}$, where the $+(-)$ sign corresponds to $\Delta I=1 / 2(3 / 2)$ amplitudes. More details on how to incorporate these $\Delta I=3 / 2$ transitions in the present mechanism can be found in Ref. [13]. It is then 
clear that, for the $\Delta I=1 / 2$ transitions considered in the present work, the correcting phase factor only affects the PV amplitudes having $|\Delta L|=1$ and $|\Delta S|=0$.

In order to account for finite size effects we include a monopole form factor at each vertex $F^{(i)}\left(\vec{q}^{2}\right)=\left(\Lambda^{(i) 2}\right.$ $\left.-\mu^{(i) 2}\right) /\left(\Lambda^{(i) 2}+\vec{q}^{2}\right)$, where the value of the cutoff $\Lambda^{(i)}$ depends on the meson $\left(\mu^{(i)}\right)$. The updated expression for the regularized potential was given in Ref. [25]. In previous calculations we used the cutoffs given by the Jülich B interaction [26]. The reason was that unlike the early $Y N$ Nijmegen model [27], which used different cutoffs depending on the irreducible representation of the baryon-baryon channel, the Jülich B was the only OBE model which used different cutoffs depending on the meson. However, the Nijmegen group has recently made available new baryon-baryon interactions in the strangeness $S=0,-1,-2,-3$, and -4 sectors [28]. These potentials are based on SU(3) extensions of the models in the $S=0$ and -1 sectors, which are fitted to the experimental data. The authors of Ref. [28] give six different models, which fit the available $N N$ and $Y N$ scattering data equally well but are characterized by different values of the magnetic vector $F /(F+D)$ ratio, ranging from 0.4447 (model NSC97a) to 0.3647 (model NSC97f). The advantage of these new models is that the form factors depend on the SU(3) type of meson. The momentum space potential for each meson is multiplied by the regularizing factor of Gaussian type $\exp \left(-\vec{q}^{2} / \Lambda^{2}\right)$, with a cutoff $\Lambda_{1}$ for the singlet meson, $\Lambda_{8}$ for the nonstrange members of the meson octet and $\Lambda_{K}$ for the strange meson. In order to accommodate to our own formalism, which uses a monopole form factor at each
TABLE I. Cutoff values in $\mathrm{MeV}$ used in the present calculation for a FF of the type $\widetilde{\Lambda}^{2} /\left(\widetilde{\Lambda}^{2}+\vec{q}^{2}\right)$, which matches the exponentialtype FF used in Ref. [28].

\begin{tabular}{lccccc}
\hline \hline$\pi$ & $\eta$ & $K$ & $\rho$ & $\omega$ & $K^{*}$ \\
\hline 1750 & 1750 & 1789 & 1232 & 1310 & 1649 \\
\hline \hline
\end{tabular}

vertex, we will match the Gaussian to a function of the type $\left[\widetilde{\Lambda}^{2} /\left(\widetilde{\Lambda}^{2}+\vec{q}^{2}\right)\right]^{2}$ at $|\vec{q}| \simeq 400 \mathrm{MeV} / c$, the most relevant momentum transfer in the $\Lambda N \rightarrow N N$ process. Since, by definition, both functional forms give 1 at $\vec{q}=0$, our alternative expression with the modified cutoffs $\widetilde{\Lambda}$, listed in Table I, gives an excellent reproduction of the Gaussian NSC97 form factors up to a momentum transfer of about $600 \mathrm{MeV} / c$.

As is well known, one of the sources of uncertainty in OBE models comes from the coupling constants between baryons and mesons. In the strong sector the different interaction models use $\mathrm{SU}(3)$ in order to obtain the $\mathrm{BBM}^{(i)}$ couplings that are not constrained experimentally. In the weak sector, only the decay of the $\Lambda$ and $\Sigma$ hyperons into nucleons and pions can be experimentally observed. For the other mesons, $\mathrm{SU}_{w}(6)$ represents a convenient tool to obtain the PV amplitudes, while for the PC ones, we use a pole model [9] with only baryon pole resonances. Details of how these coupling constants are derived can be found in Ref. [8] while the values of the $S$-wave (PV) and $P$-wave (PC) coupling constants for different parametrizations of the strong BB interaction are listed in Table II.

TABLE II. Parity conserving (PC) and parity violating (PV) weak coupling constants (CC) for different parametrizations of the strong BB interaction. The numbers are in units of $G_{F} m_{\pi}^{2}=2.21 \times 10^{-7} . V$ stands for vector coupling while $T$ stands for tensor coupling.

\begin{tabular}{|c|c|c|c|c|}
\hline & $\begin{array}{l}\text { Using strong CC } \\
\text { of NSC89 [27] }\end{array}$ & $\begin{array}{c}\text { PC }(P \text { wave }) \\
\text { Using strong CC } \\
\text { of NSC } 97 \mathrm{a}[28]\end{array}$ & $\begin{array}{l}\text { Using strong CC } \\
\text { of NSC97f [28] }\end{array}$ & $\mathrm{PV}$ ( $S$ wave) \\
\hline$\Lambda n \pi^{0}$ & 7.15 & 7.15 & 7.15 & -1.05 \\
\hline$\Lambda p \pi^{-}$ & -10.11 & -10.11 & -10.11 & 1.48 \\
\hline$\Lambda n \eta$ & -14.33 & -14.77 & -11.90 & 1.80 \\
\hline$p n K^{+}$ & -18.93 & -18.26 & -23.70 & 0.76 \\
\hline$p p K^{0}$ & 6.63 & 6.42 & 8.33 & 2.09 \\
\hline$n n K^{0}$ & -12.30 & -11.84 & -15.37 & 2.85 \\
\hline \multirow[t]{2}{*}{$\Lambda n \rho^{0}$} & (V) 3.50 & (V) 3.29 & (V) 3.29 & -1.09 \\
\hline & (T) 6.11 & (T) 7.63 & (T) 6.74 & \\
\hline \multirow[t]{2}{*}{$\left(\Lambda p \rho^{-}\right)$} & $(V)-4.94$ & $(V)-4.65$ & $(V)-4.65$ & 1.54 \\
\hline & $(T)-8.64$ & $(T)-10.79$ & $(T)-9.53$ & \\
\hline \multirow[t]{2}{*}{$(\Lambda n \omega)$} & $(V)-3.69$ & $(V)-5.97$ & $(V)-0.17$ & -1.33 \\
\hline & $(T)-8.04$ & $(T)-9.47$ & $(T)-7.43$ & \\
\hline \multirow[t]{2}{*}{$\left(p p K^{* 0}\right)$} & $(V)-4.89$ & $(V)-3.81$ & $(V)-5.46$ & 0.60 \\
\hline & (T) 9.30 & (T) 1.78 & (T) 6.23 & \\
\hline \multirow[t]{2}{*}{$\left(p n K^{*+}\right)$} & $(V)-3.61$ & $(V)-2.81$ & $(V)-4.02$ & -4.48 \\
\hline & $(T)-17.85$ & $(T)-13.40$ & $(T)-19.54$ & \\
\hline \multirow[t]{2}{*}{$\left(n n K^{* 0}\right)$} & $(V)-8.50$ & $(V)-6.62$ & $(V)-9.48$ & -3.88 \\
\hline & $(T)-8.56$ & $(T)-11.62$ & $(T)-13.31$ & \\
\hline
\end{tabular}


A measure of the amount of parity-violation in the weak decay is given by the asymmetry in the angular distribution of protons coming from the decay of polarized hypernuclei. This asymmetry is given by



where $P_{y}$ is the polarization of the hypernucleus, characteristic of the production reaction and $A_{p}$ the hypernuclear asymmetry parameter, characteristic of the weak decay. In the expression above, $J$ is the spin of the hypernucleus, $S_{y}$ is the $J$-spin operator along the direction perpendicular to the reaction plane, and $\mathcal{M}$ the hypernuclear transition amplitude. In order to compare with experiments, one has to multiply $A_{p}$ by the model dependent quantity $P_{y}$, which has to be determined theoretically for each hypernucleus [29]. By using a shell-model for the initial hypernucleus and assuming spherical configuration with no mixing, one can express this amplitude in terms of two-body transitions $\Lambda N \rightarrow N N$. The dependence of weak decay observables on the deformation of the initial ( $p$-shell) hypernucleus was investigated in Ref. [30] by means of the Nilsson model with angular momentum projection. It was found that deformation effects change these observables by about $10 \%$ from the spherical limit, a deviation that although non-negligible it is smaller than the present experimental uncertainties.

\section{EFFECTS OF THE STRONG INTERACTION}

Because of the lack of stable hyperon beams, access to the $\Delta S=-1 \Lambda N$ interaction is limited right now to the decay of hypernuclei. Hence, extracting information of the elementary weak two-body interaction requires a careful investigation of the many-body nuclear effects present in the hypernucleus.

On the one hand, one must consider that the interacting nucleon and $\Lambda$ hyperon are bound in the nucleus and they should be described by bound-state single-particle wave functions, obtained from appropriate mean-field or HartreeFock potentials. Note, however, that since the mass excess of $176 \mathrm{MeV}$ in the initial state is converted into kinetic energy of the final particles, the nucleons emerge with a large momentum of about $400 \mathrm{MeV} / \mathrm{c}$ and the decay is not very sensitive to the details of the single-particle wave function. In Ref. [24] it is shown that the decay rates obtained from various realistic $\Lambda$ wave functions differ by at most $15 \%$. On the other hand, the large momentum transfer implies that the $\Lambda N \rightarrow N N$ decay process is very sensitive to the short-range correlations induced by the strong interaction. In the initial system, one must then replace the mean-field two-particle $\Lambda N$ wave function by a correlated one that accounts for the effects of the strong $Y N$ interaction at short distances, which are not considered in mean-field models. Correlated wave functions, obtained from the soft and hard core Nijmegen $Y N$ interactions [27,31] by solving the corresponding finite nucleus scattering amplitude ( $G$ matrix) [32], were compared in Ref. [33]. The differences between the wave func- tions obtained with the two models are already significant below $0.75 \mathrm{fm}$ and give rise to decay rates that differ by slightly more than $15 \%$. However, since the present $Y N$ models are not constrained enough by the scattering data to resolve this discrepancy, we will admit this uncertainty in our initial $\Lambda N$ wave function. This uncertainty also justifies the use of a spin-independent parametrization for the $\Lambda N$ correlation function which, when multiplied with the uncorrelated one, was shown to give a decay rate in between those using the soft and hard core correlated wave functions [33].

Any realistic calculation must also take into account the fact that the two nucleons emerging from the decay feel their mutual influence, as well as that from the residual (A-2)-particle system. However, as mentioned before, the most important contribution to the decay comes from the so-called back-to-back kinematical situation, in which the two nucleons emerge with the largest possible momentum of around $400 \mathrm{MeV} / c$. For these fast moving nucleons the distortions with the residual nucleus should be small, and the importance of such effects are further diminished for inclusive observables such as the decay rates. In other words, one could take into account the interaction of the emitted nucleons with the residual nucleus through an optical potential. However, the real part should play a minor role at those high energies, and the imaginary part will remove flux from the $N N$ channel which will reappear in other multinucleon channels, such that the total strength or decay rate, which is the quantity we are interested in, will not be modified. We would face a completely different situation if we were interested in calculating the energy distribution of the nucleons, in which case one should consider these final state interaction effects, which can change the energy, direction, and charge of the primary nucleons emitted in the weak $\Lambda N \rightarrow N N$ transition, producing as well low energy secondary nucleons. The Monte Carlo simulation of Ref. [23] finds indeed that the final state interactions affect the nucleon distributions mostly at energies below $50 \mathrm{MeV}$. In the particular case of ${ }_{\Lambda}^{12} \mathrm{C}$, it is found that final state interactions produce roughly $1 / 3$ more protons at those low energies, half of which are due to charge-exchange reactions, but barely affect the distribution at higher energies and leave the decay rate intact.

Since in the present work we will only be interested in the decay rates, we will limit the treatment of final state interactions to those related to the mutual influence between the two emitted nucleons. In contrast to the strong $\Lambda N$ interaction, the nucleon-nucleon one is much better constrained by the huge amount of scattering data and, although differences in the wave function can also be observed with various potential models, they are only significant below $0.5 \mathrm{fm}$, having a moderate influence in the decay rates, as we will see.

In the literature, one finds a variety of ways of dealing with the FSI between the emitted nucleons. Some works do not include FSI, others use a phenomenological correlation function that simply multiplies the uncorrelated wave function, and others use various approximations to the $N N$ scattering equation. In the next section we will analyze the sensitivity of the non-mesonic decay observables to these various choices of FSI. The details on how the scattering $N N$ wave function is obtained from the Lippmann-Schwinger 
TABLE III. Weak decay observables for ${ }_{\Lambda}^{5} \mathrm{He}$ and ${ }_{\Lambda}^{12} \mathrm{C}$ including $\pi, \pi+K$, and all meson $\left(\pi+\eta+K+\rho+\omega+K^{*}\right)$ contributions. The total and partial nonmesonic decay rates are in units of $\Gamma_{\Lambda}$ $=3.8 \times 10^{9} \mathrm{~s}^{-1}$. The strong NSC89 coupling constants [27] and Jülich B cutoffs [26] in the (monopole) FF have been used. For the final $N N$ wave function we used the solution of a $T$-matrix equation, Eq. (A5), with the NSC93 potential model.

\begin{tabular}{lcccc}
\hline \hline${ }_{\Lambda}^{5} \mathrm{He}$ & $\Gamma_{n m}$ & $\Gamma_{n} / \Gamma_{p}$ & $\Gamma_{p}$ & $A_{p}$ \\
\hline$\pi$ & 0.438 & 0.104 & 0.397 & -0.282 \\
$\pi+K$ & 0.321 & 0.286 & 0.249 & -0.484 \\
all & 0.496 & 0.226 & 0.405 & -0.447 \\
& & & & \\
${ }_{\Lambda}^{12} \mathrm{C}$ & $\Gamma_{n m}$ & $\Gamma_{n} / \Gamma_{p}$ & $\Gamma_{p}$ & $A_{p}$ \\
\hline$\pi$ & 0.771 & 0.093 & 0.705 & 0.205 \\
$\pi+K$ & 0.558 & 0.210 & 0.461 & 0.305 \\
all & 0.834 & 0.181 & 0.706 & 0.275 \\
\hline \hline
\end{tabular}

( $T$-matrix) equation, as well as from other simplified approximations, are given in the Appendix.

\section{RESULTS AND DISCUSSION}

We start this section by showing the weak decay observables for ${ }_{\Lambda}^{5} \mathrm{He}$ and ${ }_{\Lambda}^{12} \mathrm{C}$ obtained with the model parameters used in Ref. [8], but correcting the sign in the appropriate $K$ and $K^{*}$ amplitudes, as pointed out in Sec. II. Hence, we use the coupling constants of the NSC89 strong $Y N$ interaction [27], together with a monopole form factor of the type $F\left(\vec{q}^{2}\right)=\left(\Lambda^{2}-\mu^{2}\right) /\left(\Lambda^{2}+\vec{q}^{2}\right)$ at each vertex. Each meson has a different cutoff value $\Lambda$ and, since this particular Nijmegen model distinguishes cutoffs only in terms of the transition channel, we choose, as in Ref. [8], the values of the Jülich $Y N$ interaction [26]. The results are collected in Table III and we remind the reader here of the main results given in Ref. [8] for ${ }_{\Lambda}^{12} \mathrm{C}$, namely, a nonmesonic rate of $\Gamma_{n m}=0.75 \Gamma_{\Lambda}$, where $\Gamma_{\Lambda}=3.8 \times 10^{9} \mathrm{~s}^{-1}$ is the decay rate of a free $\Lambda$, and a neutron-to-proton ratio $\Gamma_{n} / \Gamma_{p}=0.068$.

As we see, the corrected model gives a slightly larger decay rate, together with a remarkable increase in the neutron-to-proton ratio. This is due to the fact that the corrected sign gives rise to a constructive interference between the $\pi$ and $K$ contributions to the $I=1{ }^{3} S_{1} \rightarrow{ }^{3} P_{1}$ PV amplitude, instead of the destructive one found in Ref. [8] and displayed in Fig. 8 of that work. On the other hand, the incorporation of $K$ exchange to the $\pi$ mechanism lowers the PC amplitudes. This was pointed out in Fig. 7 of Ref. [8] and Figs. 4 and 5 of Ref. [34], where the contributions of all different mesons are displayed, the $\pi$ and $K$ ones showing a destructive interference in the PC amplitudes. The present constructive interference in the PV channels is especially relevant in the neutron-induced rate $\Gamma_{n}$ which lacks the PC tensor transition for $L=0$ initial states. Conversely, the proton-induced rate $\Gamma_{p}$ is dominated by the PC tensor amplitude, hence its value is lowered when $K$ exchange is added to the $\pi$ mechanism, although the reduction will now be more moderate due to the constructive interference in the less dominant PV transition. Altogether, the correct incorporation of $K$ exchange produces, in comparison to the results given in Ref. [8], a more moderate decrease in the total nonmesonic decay rate, hence a slightly larger rate is now obtained, and a $\Gamma_{n} / \Gamma_{p}$ ratio which is almost a factor of 3 larger. We note that these effects of $K$ exchange have also been pointed out in Ref. [16], where the $\pi$ and $K$ mechanisms are considered together with a description of the decay in terms of quark degrees of freedom, and in the recent work of Ref. [12], where, in addition to $\pi$ and $K$ exchange, the role of correlated two-pion exchange was also studied.

Results for the asymmetry of the emitted protons from the weak decay of polarized hypernuclei are also listed in Table III. Since this is an observable tied to the interference between PV and PC amplitudes, it will also be influenced by the change of sign in the abovementioned PV $K$ and $K^{*}$ transitions. Indeed, the magnitude of the asymmetry $A_{p}$ has increased about a factor 2 from the values given in Ref. [8] which are -0.27 for ${ }_{\Lambda}^{5} \mathrm{He}$ and 0.16 for ${ }_{\Lambda}^{12} \mathrm{C}$.

The fact that the new Nijmegen potentials [28] include different form factors depending on the meson, makes it possible to treat all the strong interaction ingredients involved in the weak decay transition $\Lambda N \rightarrow N N$ consistently within the same model. We remind the reader that the strong interaction plays a role not only in the strong vertices and form factors, but also in the PC piece of the weak vertex through the pole model and in the determination of the distorted final state $N N$ wave function. In Table IV we show the decay observables for ${ }_{\Lambda}^{5} \mathrm{He}$ and ${ }_{\Lambda}^{12} \mathrm{C}$ obtained with the models NSC97a and NSC97f.

We observe substantial differences between both models. These might come from the different coupling constants used in the strong and weak vertices of the transition $\Lambda N \rightarrow N N$ amplitude, or from the different distorted wave functions. To disentangle both effects, we show in Table $\mathrm{V}$ the results for ${ }_{\Lambda}^{5} \mathrm{He}$, obtained using the same strong interaction model for the vertices, which we choose to be the NSC97f one, and different distorted $N N$ wave functions obtained with four $N N$ interaction models, namely, the NSC97a and f [28], the NSC93 [39], and the Bonn B potential [40]. The resulting $N N$ wave functions in various relevant channels are shown in Fig. 1 for a relative momentum $p_{r}=386 \mathrm{MeV} / c$. Some minor differences appear only in the short distances, which should affect the decay rates moderately. By inspecting the results of Table $\mathrm{V}$, one can indeed see that the nonmesonic decay rate in ${ }_{\Lambda}^{5} \mathrm{He}$ varies from 0.3 to $0.4 \Gamma_{\Lambda}$, the neutron-toproton ratio $\Gamma_{n} / \Gamma_{p}$ from 0.45 to 0.5 , and the asymmetry from -0.7 to -0.6 . In conclusion, it is mainly the differences associated with the strong couplings and their influence on the weak ones that account for the differences observed in Table IV. Going back to this table, we see that there are already some differences between the results obtained with models NSC97a and NSC97f when the $K$ meson contribution is added to the $\pi$ meson one, but this cannot explain the differences in the final results when all mesons are included. Compare, for instance, the nonmesonic decay rate in ${ }_{\Lambda}^{5} \mathrm{He}$ of 0.43 obtained with the NSC97a model versus the value 0.32 obtained with the NSC97f one. In fact, by 
TABLE IV. Weak decay observables for ${ }_{\Lambda}^{5} \mathrm{He}$ and ${ }_{\Lambda}^{12} \mathrm{C}$ including $\pi, \pi+K$, and all meson $(\pi+\eta+K$ $\left.+\rho+\omega+K^{*}\right)$ contributions. The total and partial nonmesonic decay rates are in units of $\Gamma_{\Lambda}=3.8$ $\times 10^{9} \mathrm{~s}^{-1}$. The strong NSC97a (left column) and NSC97f (right column) coupling constants and cutoffs in the FF (see Table I) have been used [28]. For the final $N N$ wave function we used the solution of a $T$-matrix equation, Eq. (A5), with the corresponding NSC97a and NSC97f potential models.

\begin{tabular}{|c|c|c|c|c|c|c|c|c|}
\hline \multirow[t]{2}{*}{${ }_{\Lambda}^{5} \mathrm{He}$} & \multicolumn{2}{|c|}{$\Gamma_{n m}$} & \multicolumn{2}{|c|}{$\Gamma_{n} / \Gamma_{p}$} & \multicolumn{2}{|c|}{$\Gamma_{p}$} & \multicolumn{2}{|c|}{$A_{p}$} \\
\hline & $\mathrm{a}$ & $\mathrm{f}$ & $\mathrm{a}$ & $\mathrm{f}$ & $\mathrm{a}$ & $\mathrm{f}$ & $\mathrm{a}$ & $\mathrm{f}$ \\
\hline$\pi$ & 0.424 & 0.425 & 0.086 & 0.086 & 0.390 & 0.391 & -0.252 & -0.252 \\
\hline$\pi+K$ & 0.272 & 0.235 & 0.288 & 0.498 & 0.211 & 0.157 & -0.572 & -0.606 \\
\hline all & 0.425 & 0.317 & 0.343 & 0.457 & 0.317 & 0.218 & -0.675 & -0.682 \\
\hline $\exp$ & 0.41 & [18] & $0.93=$ & [18] & $0.21 \pm$ & $7[18]$ & $0.24 \pm$ & $22[35]$ \\
\hline \multirow[t]{2}{*}{${ }_{\Lambda}^{12} \mathrm{C}$} & \multicolumn{2}{|c|}{$\Gamma_{n m}$} & \multicolumn{2}{|c|}{$\Gamma_{n} / \Gamma_{p}$} & \multicolumn{2}{|c|}{$\Gamma_{p}$} & \multicolumn{2}{|c|}{$A_{p}$} \\
\hline & $\mathrm{a}$ & $\mathrm{f}$ & $\mathrm{a}$ & $\mathrm{f}$ & $\mathrm{a}$ & f & $\mathrm{a}$ & f \\
\hline$\pi$ & 0.762 & 0.751 & 0.078 & 0.079 & 0.707 & 0.696 & 0.169 & 0.171 \\
\hline$\pi+K$ & 0.485 & 0.413 & 0.205 & 0.343 & 0.403 & 0.308 & 0.313 & 0.320 \\
\hline all & 0.726 & 0.554 & 0.288 & 0.341 & 0.564 & 0.413 & 0.358 & 0.367 \\
\hline exp & $\begin{array}{r}1.14 \\
0.89 \pm 0 \\
1.14\end{array}$ & $\begin{array}{l}{[36]} \\
.03[19] \\
{[18]}\end{array}$ & $\begin{array}{r}1.33 \\
1.87 \pm 0 \\
0.70 \\
0.52=\end{array}$ & $\begin{array}{l}{[18]} \\
0.32[19] \\
1.00[19] \\
{[20]} \\
{[20]}\end{array}$ & $0.31_{-}^{+}$ & {$[19]$} & 0.05 & $0.53^{\mathrm{a}}$ \\
\hline
\end{tabular}

$\overline{\bar{a} \text { This number has been obtained dividing the experimental asymmetry, } \mathcal{A}=-0.01 \pm 0.10 \text { [37], by a polariza- }}$ tion of $P_{y}=-0.19$ [38].

analyzing the weak couplings listed in Table II, we can see some appreciable differences for the $N N K$ coupling constants, which are larger in the NSC97f model, thus enhancing the destructive interference of the $K$ meson and producing a reduced rate in this case. We also observe that the weak vector PC $N N \omega$ coupling constant obtained from the NSC97a model is one order of magnitude larger than that obtained from the NSC97f one. This is a consequence of a subtle cancellation in the pole model expression, which involves the difference between the strong $N N \omega$ and $\Lambda \Lambda \omega$ coupling constants. Although both models use the same strong $N N \omega$ couplings, the differences in the $\Lambda \Lambda \omega$ ones induced by the freedom allowed in the magnetic $F /(F+D)$ ratio may give rise, as is the case here, to substantial differences in the values of the PC weak coupling constants. The larger value found in the case of the NSC97a model enhances the constructive interference of the $\omega$ meson contri-

TABLE V. Weak decay observables for ${ }_{\Lambda}^{5} \mathrm{He}$ including all meson $\left(\pi+\eta+K+\rho+\omega+K^{*}\right)$ contributions. The total and partial nonmesonic decay rates are in units of $\Gamma_{\Lambda}=3.8 \times 10^{9} \mathrm{~s}^{-1}$. The strong NSC97f coupling constants and cutoffs in the FF (see Table I) have been used [28]. For the final $N N$ wave function we solve a $T$-matrix equation using different interaction models, as quoted in the table.

\begin{tabular}{lcccc}
\hline \hline${ }_{\Lambda}^{5} \mathrm{He}$ & $\Gamma_{n m}$ & $\Gamma_{n} / \Gamma_{p}$ & $\Gamma_{p}$ & $A_{p}$ \\
\hline NSC97a & 0.320 & 0.459 & 0.219 & -0.680 \\
NSC97f & 0.317 & 0.457 & 0.218 & -0.682 \\
NSC93 & 0.405 & 0.483 & 0.273 & -0.568 \\
Bonn B & 0.398 & 0.484 & 0.268 & -0.602 \\
\hline \hline
\end{tabular}

bution and explains the final difference between the decay rate obtained with the two Nijmegen models. This discussion also makes it clear that one must admit this amount of uncertainty in meson-exchange models describing the nonmesonic weak decay of hypernuclei. We should therefore conclude that the nonmesonic decay rate for ${ }_{\Lambda}^{12} \mathrm{C}$ ranges between 0.55 and $0.73 \Gamma_{\Lambda}$, the neutron to proton ratio $\Gamma_{n} / \Gamma_{p}$ between 0.29 and 0.34 , and the hypernuclear asymmetry, more stable, is 0.36 . In ${ }_{\Lambda}^{5} \mathrm{He}$, the rate obtained lies in the range $0.32-0.43 \Gamma_{\Lambda}, \Gamma_{n} / \Gamma_{p}$ in the range $0.34-0.46$, and the hypernuclear asymmetry parameter is -0.68 . We observe that our results for the nonmesonic decay rates compare satisfactorily with the experimental data, especially after considering that the size of the nonmesonic two-nucleon induced mechanism, not included here, is around $0.3 \Gamma_{\Lambda}$ for medium to heavy hypernuclei $[22,24]$. The ratio $\Gamma_{n} / \Gamma_{p}$ is considerably larger than that found in our previous works and it now lies practically within the lower side of the experimental errors. We note that a larger ratio could have been obtained, as we show below, if we had used a phenomenological treatment of FSI effects or had ignored them altogether. However, we want to stress here that the results shown in Table IV have been obtained consistently within the same strong interaction model and that involves also the dynamical generation of the corresponding $N N$ wave function through a Lippmann-Schwinger equation.

The asymmetry parameter $A_{p}$ has increased by more than a factor of 2 with respect to our previous works. As it has already been mentioned in connection to the results of Table III, this is a direct consequence of the change of sign in the $K$ and $K^{*}$ exchange PV amplitudes for transitions that do not change the spin $S$, such as $f$ in Eq. (4). Indeed, this amplitude 


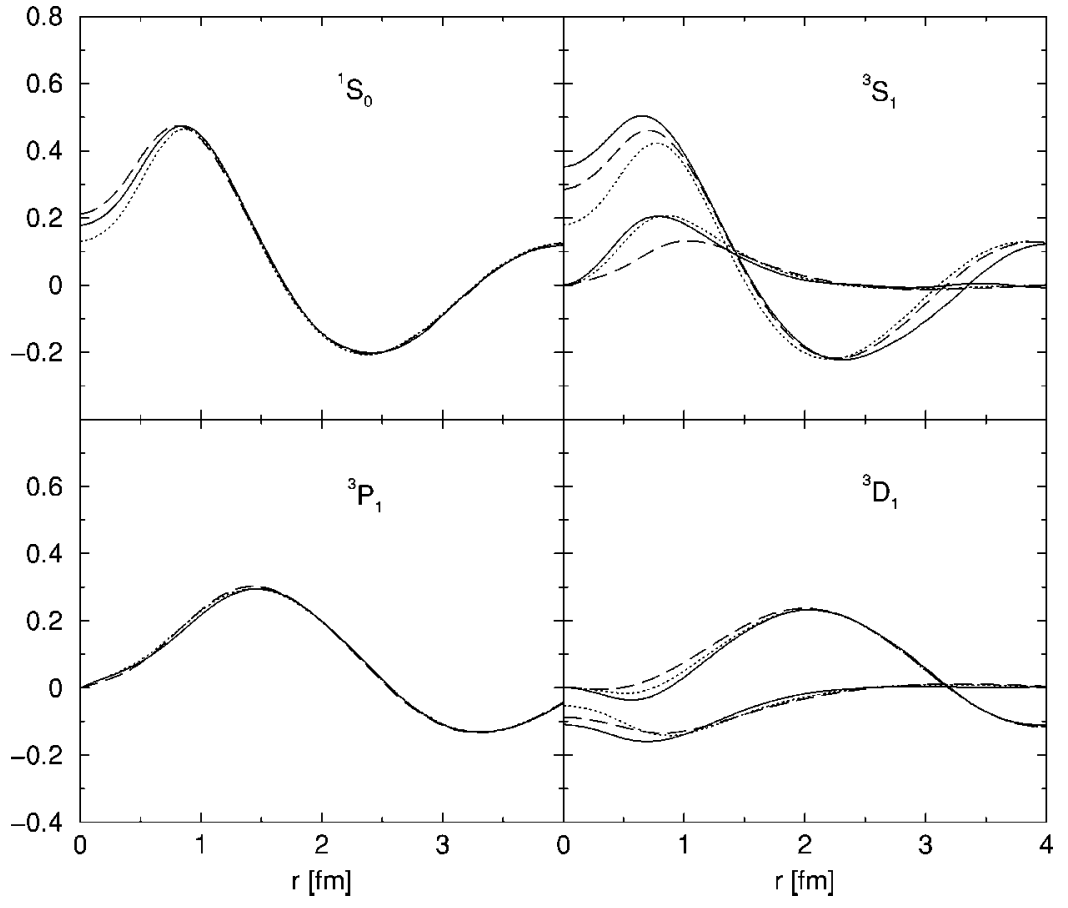

FIG. 1. $N N$ wave function in various channels for a relative momentum $p_{r}=386 \mathrm{MeV} / c$, obtained from different models of the $N N$ interaction: NSC97f [28] (solid line), NSC93 [39] (dotted line), and Bonn B [40] (dashed line). To avoid line overcrowding, only the real part of the wave function is shown in the plots. On the right-hand side panels we have also included the minority crossed component of the wave function $\left({ }^{3} S_{1}\right.$ $\rightarrow{ }^{3} D_{1}$ in the upper panel and ${ }^{3} D_{1} \rightarrow{ }^{3} S_{1}$ in the lower one). interferes with $c$ and $d$ and the magnitude of the latter tensor amplitude is especially relevant in meson-exchange models, such as the one employed here. The increased asymmetry values compare less favorably with the experimental data. While the result for ${ }_{\Lambda}^{12} \mathrm{C}$ is still within errors, the large negative value obtained here for ${ }_{\Lambda}^{5} \mathrm{He}$ is very far away from the positive small result obtained in Ref. [35]. We note that, using a weak coupling scheme, one can relate the hypernuclear asymmetry parameter $A_{p}$ to that associated to the elementary reaction $\vec{\Lambda} N \rightarrow N N, a_{\Lambda}$ [see Eq. (9) in Ref. [8]]. In the case of ${ }_{\Lambda}^{5} \mathrm{He}, a_{\Lambda}=A_{p}$ and for ${ }_{\Lambda}^{12} \mathrm{C}$ the relation is $a_{\Lambda}$ $=-2 A_{p}$. Therefore the parameter $a_{\Lambda}$ of the elementary reaction that can be extracted from our results in Table IV, turns out to be very similar in both hypernuclei. However, the present experimental results seem to contradict this finding because a small positive value is obtained for ${ }_{\Lambda}^{5} \mathrm{He}$ [35], while a large negative one was found in ${ }_{\Lambda}^{12} \mathrm{C}$ [37]. In Ref. [35] arguments are given to try to understand these differences on the basis of the fact that ${ }_{\Lambda}^{5} \mathrm{He}$ is a $s$-shell nucleus and ${ }_{\Lambda}^{12} \mathrm{C}$ a $p$-shell one, the latter one allowing transitions from initial $\Lambda N$ relative $P$-wave sates. However, it was already noticed that most of the decay for $p$-shell nuclei comes from relative $S$-wave initial states [41] and we have checked here that by removing the initial $P$-wave amplitudes, the decay rate of ${ }_{\Lambda}^{12} \mathrm{C}$ using the NSC97f model gets slightly reduced to 0.48 and the asymmetry parameter $A_{p}$ only changes to 0.34 . Hence, meson-exchange models do not explain the present experimental differences between the elementary asymmetry for ${ }_{\Lambda}^{5} \mathrm{He}$ and ${ }_{\Lambda}^{12} \mathrm{C}$, and this seems to be a new puzzle in the study of the weak decay of hypernuclei. This poses a challenge to the theoretical models and calls for further experimental efforts. In addition to valuable new information from hypernuclear weak decay experiments, it is also possible to gain direct information from the inverse reaction $p n \rightarrow p \Lambda$, already studied theoretically [25,42-44] and presently under preparation at RCNP (Osaka).

One of the purposes of this paper was to study the influence of FSI in the $\Lambda N \rightarrow N N$ transition. The results of Table $\mathrm{V}$ have already shown that using $N N$ wave functions obtained from various realistic $N N$ potentials, one obtains similar values for the weak decay observables. However, one finds in the literature several possibilitites for the treatment of FSI. They are either ignored or included through a phenomenological function, such as $f_{\text {phen }}=1-j_{0}\left(q_{c} r\right)$ with $q_{c}$ $=3.93 \mathrm{fm}^{-1}$, multiplying the free $N N$ plane wave, or through a distorted wave function built up from the $K$ matrix rather than from the $T$ matrix. In Fig. 2 we show the resulting $N N$ wave functions in various channels for a relative momentum $p_{r}=386 \mathrm{MeV} / c$, obtained from the various approaches to FSI. The solid lines represent the real and imaginary parts of the wave function obtained from the proper solution of the Lippmann-Schwinger equation ( $T$ matrix). The imaginary part is the minoritary component in all panels. The dotted line represents the result obtained when only the real part of the $T$ matrix is retained in the construction of the distorted wave function from Eq. (A10), which is further approximated by its principal part value. This was the prescription followed in Ref. [8]. The dashed line corresponds to a $K$-matrix approximation, which would be the appropriate solution for standing waves, i.e., nonpropagating solutions, as is the case in the nuclear medium, where Pauli blocking prevents two interacting nucleons of the Fermi sea from exciting intermediate $N N$ states that are already occupied by other nucleons. Finally, the dot-dashed line displays the product of the phenomenological correlation function with the corresponding noninteracting Bessel function. We observe important differences between the various approaches to the treatment of FSI, especially in the case of a phenomenological correlation function. Obviously, this will have an 


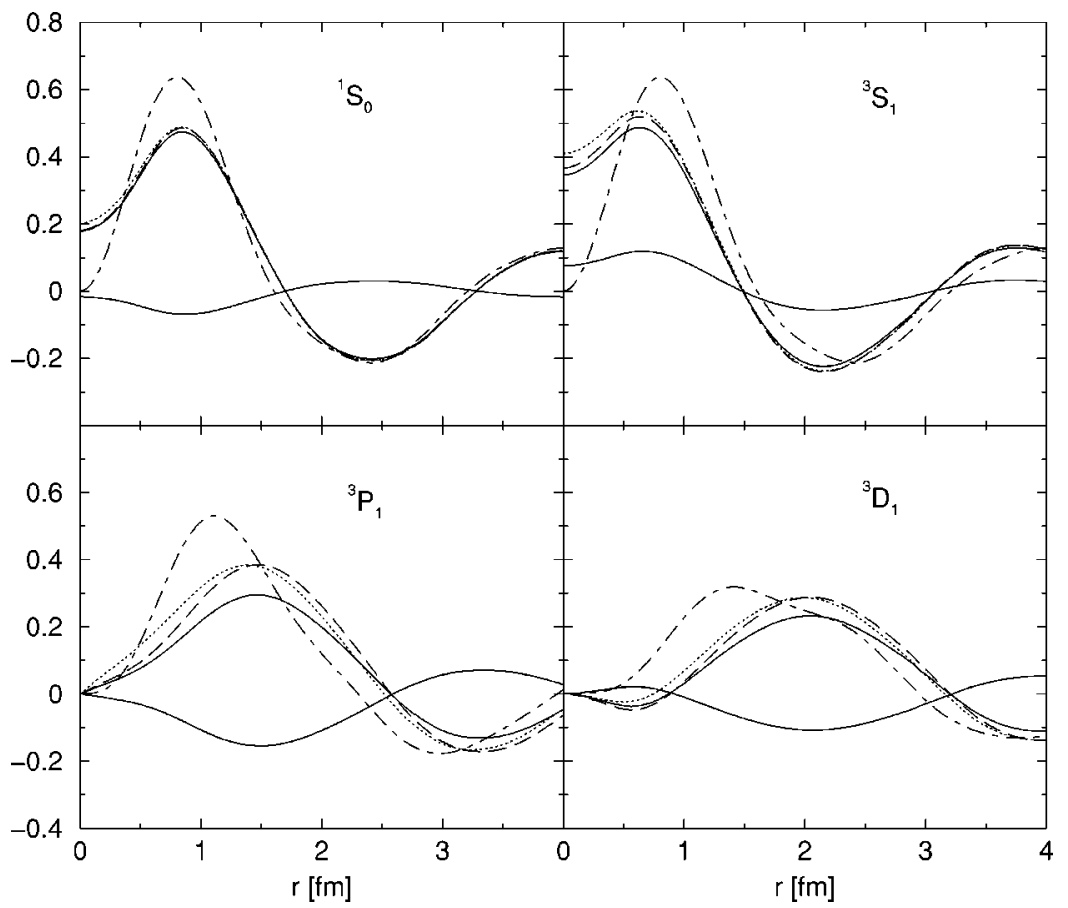

FIG. 2. $N N$ wave function in various channels for a relative momentum $p_{r}=386 \mathrm{MeV} / c$, obtained from different treatments in the solution of the scattering equation: $T$ matrix (solid lines), real part of $T$ matrix (dotted line), $K$ matrix (dashed line), and phenomenological (dot-dashed line). influence on the decay observables. As an example, we show in Table VI the results for ${ }_{\Lambda}^{5} \mathrm{He}$, obtained using the NSC97f model and taking all these different treatments for the distorted $N N$ wave functions. The rates can differ substantially, by more than a factor of 2 in some cases. The neutron-toproton ratio can also be fictitiously enhanced by using the phenomenological approach to FSI effects, or no FSI at all, while the asymmetry remains more stable.

\section{CONCLUSIONS}

We have revisited the OME model of Ref. [8] for the weak nonmesonic decay of hypernuclei to the light of the new Nijmegen baryon-baryon potentials [28]. These strong interaction models influence the weak decay mechanism, not only through the coupling constants and form factors at the strong vertex involved in the $\Lambda N \rightarrow N N$ reaction, but also via the PC piece of the weak vertex, obtained from a pole model, as well as from the corresponding correlated wave functions for the initial $\Lambda N$ and final $N N$ states.

We have corrected for a sign mistake in the $K$ - and

TABLE VI. Different approaches to the $N N$ final state wave function in the weak decay of ${ }_{\Lambda}^{5} \mathrm{He}$. The total and partial nonmesonic decay rates are in units of $\Gamma_{\Lambda}=3.8 \times 10^{9} \mathrm{~s}^{-1}$. The strong BB NSC97f model has been used.

\begin{tabular}{lcccc}
\hline \hline${ }_{\Lambda}^{5 \mathrm{He}}$ & $\Gamma_{n m}$ & $\Gamma_{n} / \Gamma_{p}$ & $\Gamma_{p}$ & $A_{p}$ \\
\hline$T$ & 0.317 & 0.457 & 0.218 & -0.682 \\
$\operatorname{Re}(T)$ & 0.490 & 0.512 & 0.324 & -0.655 \\
$K$ & 0.475 & 0.471 & 0.323 & -0.650 \\
$f_{\text {phen }}(r)$ & 0.766 & 0.619 & 0.473 & -0.671 \\
no FSI & 0.721 & 0.614 & 0.447 & -0.654 \\
\hline \hline
\end{tabular}

$K^{*}$-exchange mechanisms. This correction substantially increases the value of the neutron-to-proton ratio.

We have paid special attention to disentangle the influence of the various model ingredients involved in the decay mechanism. In particular, we have found that the uncertainties tied to the strong coupling constants are a source of uncertainty on the nonmesonic weak decay observables. Employing different strong models of the Nijmegen group, and working consistently within each one, we estimate the decay rate of ${ }_{\Lambda}^{5} \mathrm{He}$ to be in the range $0.32-0.43 \Gamma_{\Lambda}$, the neutron-toproton ratio in the range $0.34-0.46$, and the hypernuclear asymmetry parameter $A_{p}$ around -0.68 . In the case of ${ }_{\Lambda}^{12} \mathrm{C}$, the decay rate turns out to be in the range $0.55-0.73 \Gamma_{\Lambda}$, the neutron-to-proton ratio in the range $0.29-0.34$ and the asymmetry $A_{p}$ around 0.36 . The new results for the nonmesonic rates compare favorably with the present experimental data. The ratio $\Gamma_{n} / \Gamma_{p}$ has increased with respect to our previous works and it now lies practically within the lower side of the error band. The asymmetry for ${ }_{\Lambda}^{12} \mathrm{C}$ is also compatible with experiment [37] but that for ${ }_{\Lambda}^{5} \mathrm{He}$ disagrees strongly with the recent experimental observation [35]. The latter work finds a small and positive value for the elementary asymmetry parameter $a_{\Lambda}$ in ${ }_{\Lambda}^{5} \mathrm{He}$, while that for ${ }_{\Lambda}^{12} \mathrm{C}$ is large and negative. Our meson-exchange model does not explain the present experimental differences and understanding this issue is one of the current challenges, both experimental and theoretical, in the study of the weak decay of hypernuclei.

We have found a tremendous influence on the weak decay observables from the way FSI are considered, especially in the case of total and partial decay rates. A phenomenological implementation of FSI effects, or not including them at all, gives rise to decay rates that differ by more than a factor of 2 , and to a neutron-to-proton ratio about $20 \%$ larger than what is obtained with the more realistic calculation that uses the proper $N N$ scattering wave function. The differences observed in the decay rates and the $\Gamma_{n} / \Gamma_{p}$ ratio are much 
larger than the uncertainties tied to the different strong interaction models commented on above. Therefore, accurate calculations of the nonmesonic weak decay of hypernuclei demand a proper treatment of FSI effects through the solution of a $T$ matrix using realistic $N N$ interactions.

\section{ACKNOWLEDGMENTS}

The authors are indebted to K. Sasaki and M. Oka for many clarifying discussions about the inclusion of the $K$-exchange mechanism. We also thank C. Bennhold for many useful suggestions and E. Oset for discussions on the effects of an optical nucleon-nucleus potential. This work was partially supported by the U.S. Department of Energy under Grant No. DE-FG03-00-ER41132, by the DGICYT (Spain) under Contract No. PB98-1247, by the Generalitat de Catalunya Project No. SGR2000-24, and by the EEC-TMR Program EURODAPHNE under Contract No. CT98-0169.

\section{APPENDIX}

The wave function describing the relative motion of two particles moving under the influence of a two-body potential $V$ is obtained from the Lippmann-Schwinger equation

$$
\left|\Psi^{( \pm)}\right\rangle=|\Phi\rangle+\frac{1}{E-H_{0} \pm \mathrm{i} \eta} V\left|\Psi^{( \pm)}\right\rangle
$$

where $|\Phi\rangle \equiv|\vec{k}\rangle$ is the free relative motion state, the solution of the free Schrödinger equation with energy $E, H_{0}|\Phi\rangle$ $=E|\Phi\rangle$, with $H_{0}$ being the relative kinetic energy operator. The positive (negative) sign in front of the infinitesimal quantity i $\eta$ arises from the requirement that the relative motion is free in the remote past (future). Alternatively, one can write the Lippmann-Schwinger equation in terms of the transition matrix $T$, defined such that $T|\Phi\rangle=V\left|\Psi^{(+)}\right\rangle\left(\left\langle\Psi^{(-)}\right| V\right.$ $=\langle\Phi| T)$, with the result

$$
\begin{aligned}
& \left|\Psi^{(+)}\right\rangle=|\Phi\rangle+\frac{1}{E-H_{0}+\mathrm{i} \eta} T|\Phi\rangle, \\
& \left\langle\Psi^{(-)}\right|=\langle\Phi|+\langle\Phi| T \frac{1}{E-H_{0}+\mathrm{i} \eta},
\end{aligned}
$$

where the $T$ operator obeys

$$
T=V+V \frac{1}{E-H_{0}+\mathrm{i} \eta} T
$$

Let us take, for instance, $\Psi^{(-)}$(representing the two nucleons in the final state) and project Eq. (A3) into coordinate space. Inserting a complete set of states on the righthand side of Eq. (A3) and considering also the spin quantum numbers via two-particle coupled spin states, we find

$$
\begin{aligned}
\left\langle\Psi_{\vec{k}}^{(-)}, S M_{S} \mid \vec{r}\right\rangle= & \left\langle\vec{k}, S M_{S} \mid \vec{r}\right\rangle+\sum_{S^{\prime} M_{S^{\prime}}} \int d^{3} k^{\prime} \\
& \times \frac{\left\langle\vec{k}, S M_{S}|T| \vec{k}^{\prime}, S^{\prime} M_{S}^{\prime}\right\rangle\left\langle\vec{k}^{\prime}, S^{\prime} M_{S}^{\prime} \mid \vec{r}\right\rangle}{E(\vec{k})-E\left(\vec{k}^{\prime}\right)+\mathrm{i} \eta},
\end{aligned}
$$

where $\left\langle\vec{k}, S M_{S} \mid \vec{r}\right\rangle$ stands for the adjoint of the free plane wave, $\left[e^{-\mathrm{i} \vec{k} \vec{r}} /(2 \pi)^{3 / 2}\right]\left\langle S M_{S}\right|$. We perform a partial wave decomposition of the wave functions $\left\langle\Psi_{\vec{k}}^{(-)}, S M_{S} \mid \vec{r}\right\rangle$ and $\left\langle\vec{k}, S M_{S} \mid \vec{r}\right\rangle$, working in the coupled $(L S) J$ representation, and, similarly to Eq. (18.119b) of Ref. [45], we obtain

$$
\begin{aligned}
\left\langle\Psi_{\vec{k}}^{(-)}, S M_{S} \mid \vec{r}\right\rangle= & \sqrt{\frac{2}{\pi}} \sum_{L L^{\prime} S^{\prime} J M}(-\mathrm{i})^{L^{\prime}} \Psi_{L^{\prime} S^{\prime}, L S}^{(-) * J}(k, r) \\
& \times \sum_{M_{L}}\left\langle L M_{L} S M_{S} \mid J M\right\rangle Y_{L M_{L}}(\hat{k}) \mathcal{J}_{L^{\prime} S^{\prime} J}^{M \dagger}(\hat{r}),
\end{aligned}
$$

where $\mathcal{J}_{L^{\prime} S^{\prime} J}^{M \dagger}(\hat{r})$ is the adjoint of the generalized spherical harmonic given by

$$
\mathcal{J}_{L^{\prime} S^{\prime} J}^{M}(\hat{r})=\sum_{M_{L^{\prime} M_{S}^{\prime}}^{\prime}}\left\langle J M \mid L^{\prime} M_{L}^{\prime} S^{\prime} M_{S}^{\prime}\right\rangle Y_{L^{\prime} M_{L}^{\prime}}(\hat{r})\left|S^{\prime} M_{S}^{\prime}\right\rangle
$$

The partial wave decomposition for the free wave function is simply obtained by replacing in Eq. (A6)

$$
\Psi_{L^{\prime} S^{\prime}, L S}^{(-)}(k, r) \rightarrow j_{L}(k r) \delta_{L L^{\prime}} \delta_{S S^{\prime}}
$$

where $j_{L}(k r)$ is the spherical Bessel function.

A similar decomposition may be written down for the $T$-matrix elements

$$
\begin{aligned}
\left\langle\vec{k}, S M_{S}|T| \vec{k}^{\prime}, S^{\prime} M_{S}^{\prime}\right\rangle= & \sum_{J M} \sum_{L M_{L}} \sum_{L^{\prime} M_{L}^{\prime}} Y_{L M_{L}}(\hat{k}) Y_{L^{\prime} M_{L}^{\prime}}^{*}\left(\hat{k}^{\prime}\right) \\
& \times\left\langle L M_{L} S M_{S} \mid J M\right\rangle\left\langle L^{\prime} M_{L}^{\prime} S^{\prime} M_{S}^{\prime} \mid J M\right\rangle \\
& \times\left\langle k(L S) J M|T| k^{\prime}\left(L^{\prime} S^{\prime}\right) J M\right\rangle .
\end{aligned}
$$

Inserting Eqs. (A6) and (A9) into Eq. (A5), using Eqs. (A7) and (A8), and carrying out the angular integration, one obtains the following equation for the partial wave components of the correlated wave function:

$$
\begin{aligned}
\Psi_{L^{\prime} S^{\prime}, L S}^{(-) * J}(k, r)= & j_{L}(k r) \delta_{L L^{\prime}} \delta_{S S^{\prime}}+\int k^{\prime 2} d k^{\prime} \\
& \times \frac{\left\langle k(L S) J M|T| k^{\prime}\left(L^{\prime} S^{\prime}\right) J M\right\rangle j_{L^{\prime}}\left(k^{\prime} r\right)}{E(k)-E\left(k^{\prime}\right)+\mathrm{i} \eta},
\end{aligned}
$$


where the partial wave $T$-matrix elements fulfill the integral equation

$$
\begin{aligned}
\left\langle k(L S) J M|T| k^{\prime}\left(L^{\prime} S^{\prime}\right) J M\right\rangle & \\
= & \left\langle k(L S) J M|V| k^{\prime}\left(L^{\prime} S^{\prime}\right) J M\right\rangle \\
& +\sum_{S^{\prime \prime} L^{\prime \prime}} \int k^{\prime \prime} 2 d k^{\prime \prime} \frac{\left\langle k(L S) J M|V| k^{\prime \prime}\left(L^{\prime \prime} S^{\prime \prime}\right) J M\right\rangle}{E(k)-E\left(k^{\prime \prime}\right)+\mathrm{i} \eta} \\
& \times\left\langle k^{\prime \prime}\left(L^{\prime \prime} S^{\prime \prime}\right) J M|T| k^{\prime}\left(L^{\prime} S^{\prime}\right) J M\right\rangle .
\end{aligned}
$$

Both equations (A10) and (A11) are solved in momentum space following a numerical matrix inversion method described in Ref. [46]. Isospin is easy to incorporate in the final step of our calculation by multiplying the resulting correlated wave function by the isospinor $\chi_{T}^{M_{T}}$, allowing only for those cases that fulfill the antisymmetry requirement $L+S+T$ = odd.

An alternative but approximate way of implementing the
$N N$ final state interactions is through the $K$-matrix operator, which obeys

$$
K=V+V \mathcal{P}\left(\frac{1}{E-H_{0}}\right) K
$$

where $\mathcal{P}$ indicates the Cauchy principal value. Standing waves can then be obtained by following the same procedure of Eqs. (A5) through (A11) but replacing the $T$-matrix elements by those of the real $K$ matrix.

Finally, a simple way to include the effects of the strong interaction between the particles is to use a phenomenological correlation function $f_{\text {phen }}(r)$, such that

$$
\Psi_{L^{\prime} S^{\prime}, L S}^{(-) * J}(k, r)=f_{\text {phen }}(r) j_{L}(k r) \delta_{L L^{\prime}} \delta_{S S^{\prime}} .
$$

This has been the approach followed by several authors, and functions such as a Gaussian or of the type $f_{\text {phen }}(r)$ $=1-j_{0}\left(q_{c} r\right)$ with $q_{c}=3.93 \mathrm{fm}^{-1}$ have been used [16].
[1] M.M. Block and R.H. Dalitz, Phys. Rev. Lett. 11, 96 (1963); R.H. Dalitz and G. Rajasekharan, Phys. Lett. 1, 58 (1962).

[2] J. Cohen, Progress Particle Nuclear Physics (Pergamon, New York, 1990), p. 139.

[3] B.F. Gibson and E.V. Hungerford, Phys. Rep. 257, 349 (1995).

[4] E. Oset and A. Ramos, Prog. Part. Nucl. Phys. 41, 191 (1998).

[5] J.B. Adams, Phys. Rev. 156, 1611 (1967).

[6] E. Oset and L.L. Salcedo, Nucl. Phys. A443, 704 (1985).

[7] B.H.J. McKellar and B.F. Gibson, Phys. Rev. C 30, 322 (1984).

[8] A. Parreño, A. Ramos, and C. Bennhold, Phys. Rev. C 56, 339 (1997).

[9] J.F. Dubach, G.B. Feldman, B.R. Holstein, and L. de la Torre, Ann. Phys. (N.Y.) 249, 146 (1996); L. de la Torre, Ph.D. thesis, University of Massachusetts, 1982.

[10] K. Itonaga, T. Ueda, and T. Motoba, Nucl. Phys. A585, 331c (1995); Weak and Electromagnetic Interactions in Nuclei, edited by H. Ejiri, T. Kishimoto, and T. Sato (World Scientific, Singapore, 1995), p. 546; Nucl. Phys. A639, 329 (1998).

[11] M. Shmatikov, Phys. Lett. B 322, 311 (1994); Nucl. Phys. A580, 538 (1994).

[12] D. Jido, E. Oset, and J.E. Palomar, Nucl. Phys. A694, 525 (2001).

[13] A. Parreño, A. Ramos, C. Bennhold, and K. Maltman, Phys. Lett. B 435, 1 (1998).

[14] C.-Y. Cheung, D.P. Heddle, and L.S. Kisslinger, Phys. Rev. C 27, 335 (1983); D.P. Heddle and L.S. Kisslinger, ibid. 33, 608 (1986).

[15] T. Inoue, S. Takeuchi, and M. Oka, Nucl. Phys. A597, 563 (1996); T. Inoue, M. Oka, T. Motoba, and K. Itonaga, ibid. A633, 312 (1998).

[16] K. Sasaki, T. Inoue, and M. Oka, Nucl. Phys. A669, 331 (2000); A678, 455 (2000).

[17] K. Maltman and M. Shmatikov, Phys. Lett. B 331, 1 (1994).

[18] J.J. Szymanski et al., Phys. Rev. C 43, 849 (1991).

[19] H. Noumi et al., Phys. Rev. C 52, 2936 (1995).
[20] A. Montwill et al., Nucl. Phys. A234, 413 (1974).

[21] W.M. Alberico, A. De Pace, M. Ericson, and A. Molinari, Phys. Lett. B 256, 134 (1991).

[22] A. Ramos, E. Oset, and L.L. Salcedo, Phys. Rev. C 50, 2314 (1994).

[23] A. Ramos, M.J. Vicente-Vacas, and E. Oset, Phys. Rev. C 55, 735 (1997).

[24] W.M. Alberico, A. De Pace, G. Garbarino, and A. Ramos, Phys. Rev. C 61, 044314 (2000).

[25] A. Parreño, A. Ramos, N.G. Kelkar, and C. Bennhold, Phys. Rev. C 59, 2122 (1999).

[26] B. Holzenkamp, K. Holinde, and J. Speth, Nucl. Phys. A500, 485 (1989).

[27] P.M.M. Maessen, Th. A. Rijken, and J.J. de Swart, Phys. Rev. C 40, 2226 (1989).

[28] V.G.J. Stoks and Th. A. Rijken, Phys. Rev. C 59, 3009 (1999); Th. A. Rijken, V.G.J. Stoks, and Y. Yamamoto, ibid. 59, 21 (1999).

[29] K. Itonaga, T. Motoba, O. Richter, and M. Sotona, Phys. Rev. C 49, 1045 (1994).

[30] K. Hagino and A. Parreño, Phys. Rev. C 63, 044318 (2001).

[31] M.M. Nagels, T.A. Rijken, and J.J. de Swart, Phys. Rev. D 15, 2547 (1977).

[32] D. Halderson, Phys. Rev. C 48, 581 (1993).

[33] A. Parreño, A. Ramos, C. Bennhold, and D. Halderson, in Dynamical Features of Nuclei and Finite Fermi Systems (World Scientific, Singapore, 1994), p. 318.

[34] J. Golak, K. Miyagawa, H. Kamada, H. Witala, W. Glöckle, A. Parreño, A. Ramos, and C. Bennhold, Phys. Rev. C 55, 2196 (1997).

[35] S. Ajimura et al., Phys. Rev. Lett. 18, 4052 (2000).

[36] H. Bhang et al., Phys. Rev. Lett. 81, 4321 (1998).

[37] S. Ajimura et al., Phys. Lett. B 282, 293 (1992).

[38] A. Ramos, E. van Meijgaard, C. Bennhold, and B.K. Jennings, Nucl. Phys. A544, 703 (1992).

[39] V.G.J. Stoks, R.A.M. Klomp, C.P.F. Terheggen, and J.J. de 
Swart, Phys. Rev. C 49, 2950 (1994).

[40] R. Machleidt in Computational Nuclear Physics. 2. Nuclear Reactions, edited by K. Langanke, J.A. Maruhn, and S.E. Koonin (Springer-Verlag, Berlin, 1993), pp. 1-29.

[41] C. Bennhold and A. Ramos. Phys. Rev. C 45, 3017 (1992).

[42] J. Haidenbauer, K. Holinde, K. Kilian, T. Sefzick, and A.W. Thomas, Phys. Rev. C 52, 3496 (1994).
[43] H. Nabetani, T. Ogaito, T. Sato, and T. Kishimoto, Phys. Rev. C 60, 017001 (1999).

[44] T. Inoue, K. Sasaki, M. Oka, and S. Takeuchi, Prog. Theor. Phys. Suppl. 137, 147 (2000).

[45] C.J. Joachain, Quantum Collision Theory (Elsevier, Amsterdam, 1975).

[46] M.I. Haftel and F. Tabakin, Nucl. Phys. A158, 1 (1970). 